\title{
We are falling behind on TB elimination targets: can whole-genome sequencing guide our efforts?
}

\author{
Nicole Wheeler ${ }^{\oplus}$
}

TB remains a leading cause of morbidity and mortality worldwide, despite international eradication efforts. The WHO's 'End Tuberculosis Strategy' set the ambitious goal of achieving a $90 \%$ reduction in the incidence by the year 2035; yet, we are currently only achieving a $2 \%$ reduction per year. ${ }^{1}$ There is a pressing need to develop more effective control strategies in order to meet this target. A major challenge to eradication efforts is the increasing proportion of TB infections that are resistant to antibiotics. ${ }^{2}$ Approximately half a million new cases of multidrug-resistant TB (MDR-TB) emerge each year, and of these, approximately $8.5 \%$ are classed as extensively drug-resistant (XDR). ${ }^{2}$ Because MDR-TB is difficult to treat at the individual level, targeting the key drivers of this increase is important for curbing resistance. To do this, we need a clear understanding of what these key drivers are.

Identifying drug resistance in TB cases is challenging, due to the expense of diagnosis, the time required for culture-based methods (weeks to months) and the lack of standardisation of testing methods for some second-line drugs. ${ }^{3}$ This delay in identification prolongs the opportunity for these strains to transmit to others. Whole-genome sequencing (WGS) has improved in cost and throughput, allowing an increase in its use. ${ }^{4}$ Resistance emerges predominantly through mutation, ${ }^{5}$ making TB a relatively simple paradigm for WGS identification of resistance. Thanks to a growing understanding of the mechanisms underlying resistance, we are now able to detect most MDR-TB (87\%-89\%) and XDR-TB (77\%-79\%) using WGS data. ${ }^{5}$ In line with these advances, large genomic collections of TB spanning different countries are being released, and used to study resistance. ${ }^{6}$ In this issue of Thorax, Cohen et $a l^{7}$ explore the value of combining these large collections to trace the global

Centre for Genomic Pathogen Surveillance, Wellcome Sanger Institute, Cambridge, UK

Correspondence to Dr Nicole Wheeler, Centre for Genomic Pathogen Surveillance, Wellcome Sanger Institute, Cambridge CB10 1SA, UK; nw17@sanger.ac.uk emergence and spread of MDR-TB. This study collated WGS data from 5310 isolates from 15 separate studies, spanning 48 countries and 5 continents. Their work leads on from a previous study in which this data was used to identify 573 independent emergences of MDR, and the mutations underpinning these. ${ }^{6}$ Here, the authors focus on the movement of these MDR strains globally.

A key gap in our knowledge is the relative contribution of de novo emergence and transmission of resistant strains to the burden of resistance. Understanding this balance is critical to providing the best interventions to reduce resistance and TB burden. If de novo resistance dominates, then effectively treating susceptible cases should be a priority; however, if transmission dominates, then more resources should be directed at correctly identifying MDR cases and treating them quickly and effectively. ${ }^{8}$ This balance is expected to shift over time, as drug-resistant strains become more prevalent.

Evidence of the large contribution of local transmission to the overall burden of resistance is growing steadily, ${ }^{9}{ }^{10}$ but we still have a limited understanding of the impact of global transmission. In this study, 360 of the 573 MDR strains identified consisted of a single isolate, providing no evidence for transmission, while 195 showed evidence of emerging and transmitting between at least two individuals. A total of 32 of these clades were found in multiple countries, consistent with global spread. The median estimated origin of these globally dispersed clades was between 13 and 27 years ago. This phenomenon had been captured previously in smaller snapshots, ${ }^{11}$ but not with such a large and geographically diverse collection of samples. In nearly all incidents of international spread, the isolates from different countries came from different investigations and the links between them would not have been identified by any previous study.

One area for further research highlighted by the authors was whether there are factors that distinguish MDR strains that spread around the world from those that don't. Previously, particular genetic backgrounds, such as lineage 2 (Beijing strains), ${ }^{11}{ }^{12}$ low-cost resistance mutations $^{13}$ and compensatory mutations, such as mutations in rpo $A$ and $r p o C$, which compensate for mutation in $r p o B,{ }^{14}$ have allowed MDR strains to achieve transmission rates similar to or even greater than susceptible strains. Differentiating genetic factors could clarify conflicting evidence as to whether increased drug resistance helps or hinders transmission. For example, in two studies conducted in similar regions of Peru, a 2010-2013 prospective study found MDR-TB strains transmitted to household contacts half as frequently as drug-susceptible strains, ${ }^{15}$ while a 1996-2003 retrospective study found that XDR-TB strains transmitted to household contacts at nearly twice the rate of MDR-TB strains. ${ }^{16}$ In this study, the highly successful Beijing strain accounted for over half of the observed migration events; however, global spread was also observed in rarer strains. Notably, no instances of the global spread of XDR strains were observed, but XDR cases emerged in over half of the globally disseminated MDR clades. It is unclear whether this indicates that XDR strains have an impaired ability to spread globally, or whether this was due to their rarity in this collection.

The incidence of TB and the outcome of treatment efforts varies markedly by country. A total of 30 countries identified by the WHO as high-burden accounted for $87 \%$ of all cases in 2017, while Europe and the Americas accounted for $6 \%$ of cases. ${ }^{2}$ Each of the main TB lineages appears to associate predominantly with one region; however, Cohen et al observed that global migration events often involved both high-burden and low-burden countries. They inferred that this resistance most likely emerged in high-burden settings and migrated to low-burden settings, but were not able to prove this definitively. The migrant clades were very small and were likely to be affected by sampling bias, meaning accurate inference of the direction of movement was not possible. The authors also acknowledge that these transmission events may have involved intermediate movement that was not sampled. The amount of migration between countries tended to reflect how well-sampled each country was, suggesting that better sampling of under-represented regions could reveal more migratory clades.

Global analysis of important pathogens can yield key insights into pathogen dynamics, but is reliant on representative sampling. Currently, one-third of the world's population is estimated to 
be infected with Mycobacterium tuberculosis. ${ }^{2}$ Thus, this collection of around 5000 samples provides only a small window into the global diversity of this disease. In addition, the bias in sampling strategies and locations in each of the studies that contributed WGS data to this analysis will cause this investigation to give an incomplete and potentially slanted picture of the global emergence and movement of resistance.

The distribution of TB in this study did not match the reported distribution of $\mathrm{TB}$ worldwide, ${ }^{6}$ and rates of resistance in this data set were higher than documented global rates (18\% compared with 5.5\%), ${ }^{2}$ reflecting predictable biases in previous sampling efforts. The authors noted that South America, Asia and the Pacific Islands were all poorly represented in past sequencing efforts, indicating a need for more targeted work in these previously neglected regions. This pattern is consistent across large international collections of pathogen sequences. ${ }^{17}$ This may be due to high-income countries sequencing a disproportionately high number of isolates relative to the burden of disease, and low-income countries with high burden of disease being targeted with additional sequencing, leaving countries of intermediate income and burden underserved by both approaches.

This investigation of previously collected samples did not achieve the balance and resolution required to accurately establish the balance between de novo and transmitted MDR, and the directionality of movement of internationally mobile strains. However, the results indicate that MDR-TB has emerged independently multiple times, and that strains from diverse genetic backgrounds are capable of not only transmitting between individuals but also of travelling vast distances and establishing in new countries. Coordinated efforts to generate and share genomic data for key pathogens will allow us to gain even more insight into these dynamics. However, thought needs to be given to the allocation of resources and effort. Routine implementation of sequencing in high-income, low-burden countries will not fill the major gaps identified in this data. If anything, this will further exacerbate the divide between our understanding and management of TB transmission in high-income and low-income countries, and potentially miss lineages that make large contributions to the global burden of disease. To truly capture the dynamics of resistance, sampling would need to be larger, globally representative, and collected in a structured manner. ${ }^{18}$ However, implementing routine WGS in low-resource settings poses an array of costs and challenges. ${ }^{19}$ More representative sampling will require international collaboration and better global investment into surveillance infrastructure and training.

The authors highlight a critical role for global surveillance and data sharing for gaining a better understanding of international transmission of MDR-TB. They also demonstrate the value that can be gained by pooling and analysing data from previously published analyses. This work illustrates the insight that WGS data can provide into what drives the emergence and spread of resistance, and highlights the need for better efforts to collect globally representative data in the future.

\section{Contributors NW drafted and reviewed the article.}

Funding NW is supported by National Institutes of Health grant U01CA207167. The contents of this paper are solely the responsibility of the author and do not necessarily represent the official views of the National Institutes of Health.

Competing interests None declared.

Patient consent for publication Not required.

Provenance and peer review Commissioned; externally peer reviewed.

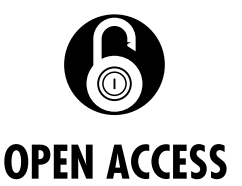

Open access This is an open access article distributed in accordance with the Creative Commons Attribution Non Commercial (CC BY-NC 4.0) license, which permits others to distribute, remix, adapt, build upon this work non-commercially, and license their derivative works on different terms, provided the original work is properly cited, appropriate credit is given, any changes made indicated, and the use is non-commercial. See: http:// creativecommons.org/licenses/by-nc/4.0/.

(c) Author(s) (or their employer(s)) 2019. Re-use permitted under CC BY-NC. No commercial re-use. See rights and permissions. Published by BMJ.

- Additional material is published online only. To view, please visit the journal online (http://dx.doi.org/ 10.1136/thoraxjnl-2019-213098).

\section{Check for updates}

To cite Wheeler N. Thorax 2019;74:833-834.

Accepted 5 July 2019

Published Online First 22 July 2019

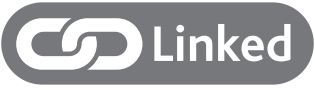

http://dx.doi.org/10.1136/thoraxjnl-2018-211616
Thorax 2019;74:833-834.

doi:10.1136/thoraxjnl-2019-213098

\section{REFERENCES}

1 Uplekar M, Weil D, Lonnroth K, et al. Who's new end TB strategy. The Lancet 2015:385:1799-801.

2 World Health Organisation. Global tuberculosis report 2018, 2018. Available: https://www.who.int/tb/ publications/global_report/en/

3 World Health Organisation. Guidelines for surveillance of drug resistance in tuberculosis. 5th Edition, 2015.

4 Walker TM, Cruz ALG, Peto TE, et al. Tuberculosis is changing. Lancet Infect Dis 2017;17:359-61.

5 Coll F, Phelan J, Hill-Cawthorne GA, et al. GenomeWide analysis of multi- and extensively drugresistant Mycobacterium tuberculosis. Nat Genet 2018;50:307-16.

6 Manson AL, Cohen KA, Abeel T, et al. Genomic analysis of globally diverse Mycobacterium tuberculosis strains provides insights into the emergence and spread of multidrug resistance. Nat Genet 2017;49:395-402.

7 Cohen KA, Manson AL, Abeel T, et al. Extensive global movement of multidrug-resistant $M$. tuberculosis strains revealed by whole-genome analysis. Thorax 2019;74:881-8

8 Dheda K, Gumbo T, Maartens G, et al. The epidemiology, pathogenesis, transmission, diagnosis, and management of multidrug-resistant, extensively drug-resistant, and incurable tuberculosis. Lancet Respir Med 2017;5:291-360.

9 Kendall EA, Fofana MO, Dowdy DW. Burden of transmitted multidrug resistance in epidemics of tuberculosis: a transmission modelling analysis. Lancet Respir Med 2015;3:963-72.

10 Yang C, Luo T, Shen X, et al. Transmission of multidrugresistant Mycobacterium tuberculosis in Shanghai, China: a retrospective observational study using whole-genome sequencing and epidemiological investigation. Lancet Infect Dis 2017;17:275-84.

11 Merker M, Blin C, Mona S, et al. Evolutionary history and global spread of the Mycobacterium tuberculosis Beijing lineage. Nat Genet 2015;47:242-9.

12 Devaux I, Kremer K, Heersma H, et al. Clusters of multidrug-resistant Mycobacterium tuberculosis cases, Europe. Emerg Infect Dis 2009;15:1052-60.

13 van Doorn HR, de Haas PEW, Kremer K, et al. Public health impact of isoniazid-resistant Mycobacterium tuberculosis strains with a mutation at amino-acid position 315 of katG: a decade of experience in the Netherlands. Clin Microbiol Infect 2006;12:769-75.

14 Casali N, Nikolayevskyy V, Balabanova Y, et al. Evolution and transmission of drug-resistant tuberculosis in a Russian population. Nat Genet 2014;46:279-86.

15 Grandjean L, Gilman RH, Martin L, et al. Transmission of multidrug-resistant and drug-susceptible tuberculosis within households: a prospective cohort study. PLoS Med 2015;12:e1001843.

16 Becerra MC, Appleton SC, Franke MF, et al. Tuberculosis burden in households of patients with multidrug-resistant and extensively drug-resistant tuberculosis: a retrospective cohort study. The Lancet 2011;377:147-52.

17 Centre for Genomic Pathogen Surveillance. Pathogenwatch map of available genome collections. Available: https://pathogen.watch/genomes/all/map

18 Grundmann H, Schouls LM, Aanensen DM, et al. The dynamic changes of dominant clones of Staphylococcus aureus causing bloodstream infections in the European region: results of a second structured survey. Euro Surveill 2014;19.

19 Helmy M, Awad M, Mosa KA. Limited resources of genome sequencing in developing countries: challenges and solutions. App/ Trans/ Genom 2016:9:15-19. 